\title{
Thermocapillary Flow in a J et of Liquid Film Painted on a Moving Boundary
}

\author{
W. R. Hu*,t,‡ and N. Imaishi ${ }^{\ddagger}$ \\ Institute of Mechanics, Chinese Academy of Science, Beijing 100080, China, and Institute of \\ Advanced Material Study, Kyushu University, Kasuga, Fukuoka 816-8580, J apan
}

Received December 2, 1999. In Final Form: J anuary 18, 2000

\begin{abstract}
In the present paper, a liquid (or melt) film of relatively higher temperature ejected from a vessel and painted on the moving solid boundary is analyzed. The thermocapillary flow is driven by the temperature gradient on the free surface of a liquid film, because of the heat transfer from the liquid with higher temperaturetotheenvironmental gas with relatively lower temperature. Thethermocapillary flow changes the height profile of the liquid film. The analysis is based on the approximations of lubrication theory and perturbation theory, and the equation of liquid height and the process of thermal hydrodynamics in the liquid film are solved for a given temperature distribution on the solid boundary.
\end{abstract}

\section{Introduction}

The processing of film and polymer requires the understanding of the hydrodynamic process, such as the temperature, pressure, flow field, and diameter or height distributions; see, for example, refs 1-3. Thetemperature of the liquid or the melt, ejected from the nozzle of a manufacture vessel, is relatively higher in comparison with theenvironmental gas temperature, and then, there is a strong heat exchange, especially in the region near the exit of vessel. The heat transfer from the liquid film to the environmental gas forms a temperature gradient and then the surface tension gradient on the free surface and induces thermocapillary flow, which will, of course, changethecross section of the jet liquid. The Barus effect or Die Swell effect on the change of cross section in a polymer jet is often observed in the processing and is explained usually by therheol ogy property of the polymer melt. However, there are many suggestions on the viscoelastic constitutive relationships and the rheology properties of the polymer, and suitable and acceptable descriptions on the relationships and properties are still open problems that need to be studied in the future. ${ }^{4}$

It is known that thermocapillary flow is induced in a thin liquid layer which lays on a solid wall with nonuniform temperature distribution and the heat transfer process will result the nonuniformity of the liquid thickness. By using theapproximation of lubrication theory, an ordinary differential equation of liquid thickness and its solution for given boundary temperature were obtained for unsteady cases in a thin liquid layer. ${ }^{5} \mathrm{~A}$ similar method was applied to discuss the steady cases where a smooth condition at the symmetric cross section was improved. ${ }^{6}$

* To whom correspondence should be addressed at the Chinese Academy of Science.

${ }^{\dagger}$ Chinese Academy of Science.

‡ Kyushu University.

(1) Tucker, C. L. Computer Modeling for Polymer Processing; Hanser: New York, 1989.

(2) Kennedy, P. Flow Analysis of Ejection Models; Hanser: New York, 1995.

(3) Silagy, D.; Demay, Y.; Agussant, J . F. Stationary and stability analysis of the film casting process. J. Non-Newtonian Fluid Mech. 1998, 79, 563.

(4) Leonov, A. I. Visoel astic constitutive equations and rheol ogy for high-speed polymer processing. Polym. Int. 1995, 36, 187.

(5) Pimputkar, S. M.; Ostrach, S. Transient thermocapillary flow in the thin liquid layers. Phys. Fluids 1980, 23, 1281.

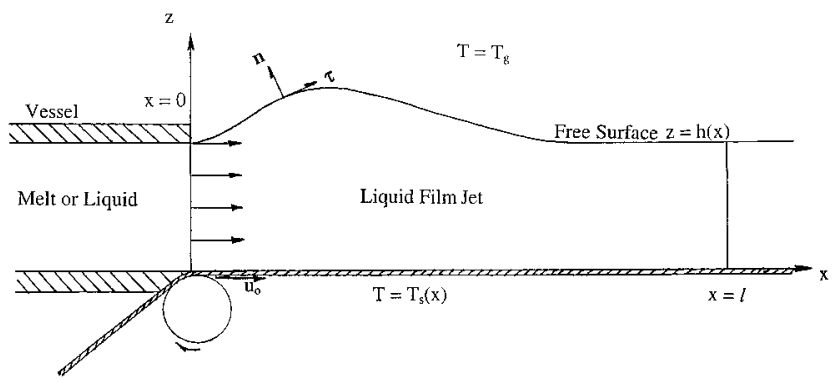

Figure 1. Schematic diagram of the physical model of a jet liquid film.

The problem of motionless and infinitely extending solid boundary was studied in refs 5 and 6 . Furthermore, the solutal capillary flow induced by the surfactant of the liquid may increase the thickness of the liquid layer. ${ }^{7}$

In the present paper, the change of cross section due to thethermocapillary convection in a two-dimensional and steady model is discussed for the case of jet liquid film painting on a moving solid boundary, and the similar approximation of lubrication theory is assumed. In the case of a small geometrical aspect ratioand small capillary number, the perturbation method is applied. The firstorder solutions of the problem can beobtained analytically, and a fourth-order ordinary differential equation of the liquid layer height can be demonstrated. Four boundary conditions aregiven to solvethe equation of liquid height, and thesolution clearly shows the effect of thermocapillary convection on the thickness variation of the liquid layer. The conclusion of this paper suggests that a large enlargement of the cross section, which is usually explained by therheol ogy property of theliquid, may bedue to the thermocapillary effect in the liquid jet of a Newtonian fluid.

\section{Model of J et Liquid Film}

A simplified model is suggested as shown in Figure 1 , where the liquid layer with the height $h_{0}$ is ejected from a nozzle of a melt or liquid vessel and then attached to a

(6) Hitt, D. L.; Smith, M. K. Radiation-driven thermocapillary flows in optically thick liquid films. Phys. Fluids 1993, A5, 2624.

(7) Ramdaul, O. O.; Quere, D. Thickening Factor in Marangoni Coating. Langmuir 1997, 13, 2911. 
moving solid film. This model avoids the complexity in detail ed proceedings and emphasizes theinfluence of the thermocapillary convection on the height variation. The Cartesian coordinate system $(x, y, z)$ is adopted, the problem is assumed two-dimensional, and $\partial / \partial y=0$. The melt or liquid flows in a distance I far from the exit of the vessel, and there is a small geometrical aspect ratio $\epsilon=$ $\mathrm{h}_{\mathrm{d}} \mathrm{l} \ll 1$. The liquid temperature $\mathrm{T}_{\mathrm{e}}$ at the vessel exit is higher than the environmental gas temperature $T_{g}$ and thetemperature $T_{1}$ at $x=I$. A moving solid film is touched smoothly with thelower boundary of thevessel and moves with the same velocity $u_{0}$ of the liquid at the lower boundary of the vessel exit. This assumption idealizes the model to avoid mathematical complexity at the contact and is beneficial to analyze the thermocapillary effects. The heat transfer between moving solid film, liquid jet, and environmental gas results in a large temperature gradient on the free surface, especially near the exit of the vessel, and then, the thermocapillary flow will be driven in addition to the jet flow.

The liquid is assumed to be incompressible and Newtonian with constant kinematics viscosity $v$ and thermal diffusivity $\kappa$. The relationships of the mass conservation, themomentum conservation, and theenergy conservation will be given as

$$
\begin{gathered}
\frac{\partial u}{\partial x}+\frac{\partial w}{\partial z}=0 \\
u \frac{\partial u}{\partial x}+w \frac{\partial u}{\partial z}=-\frac{1}{\rho} \frac{\partial p}{\partial x}+v\left(\frac{\partial^{2} u}{\partial x^{2}}+\frac{\partial^{2} u}{\partial z^{2}}\right) \\
u \frac{\partial w}{\partial x}+w \frac{\partial w}{\partial z}=-\frac{1}{\rho} \frac{\partial p}{\partial z}+v\left(\frac{\partial^{2} w}{\partial x^{2}}+\frac{\partial^{2} w}{\partial z^{2}}\right) \\
u \frac{\partial T}{\partial x}+w \frac{\partial T}{\partial z}=\kappa\left(\frac{\partial^{2} T}{\partial x^{2}}+\frac{\partial^{2} T}{\partial z^{2}}\right)
\end{gathered}
$$

where $(u, 0, w)$ is the velocity vector and $\rho, p$, and $T$ are respectively the density, pressure, and temperature of the liquid.

The boundary condition of the liquid layer may be summarized as follows:

$z=0$ :

$$
\mathrm{u}=\mathrm{u}_{\mathrm{o}} \quad \mathrm{w}=0 \quad \mathrm{~T}=\mathrm{T}_{\mathrm{s}}(\mathrm{x})
$$

$z=h(x):$

$$
\begin{gathered}
u \frac{d h}{d x}=w \\
\frac{\rho v}{\left(1+h^{\prime 2}\right)}\left[\left(1-h^{\prime 2}\right)\left(\frac{\partial w}{\partial x}-\frac{\partial u}{\partial z}\right)+2 h^{\prime}\left(\frac{\partial w}{\partial z}-\frac{\partial u}{\partial x}\right)\right]= \\
\sigma_{\top}^{\prime}\left(\frac{\partial T}{\partial x}+h^{\prime} \frac{\partial T}{\partial z}\right) \\
p=\frac{2 \rho v}{1+h^{\prime 2}}\left[\left(\frac{\partial w}{\partial z}-h^{\prime} \frac{\partial u}{\partial x}\right)-h^{\prime}\left(\frac{\partial w}{\partial x}+h^{\prime} \frac{\partial u}{\partial x}\right)\right]- \\
{\left[\sigma_{o}+\sigma_{T}^{\prime}\left(T-T_{*}\right)\right] \frac{h^{\prime \prime}}{\left(1+h^{\prime 2}\right)^{3 / 2}}} \\
k \frac{\partial T}{\partial n}=-H\left(T-T_{g}\right)
\end{gathered}
$$

$\mathrm{x}=0$ :

$$
\mathrm{u}=\mathrm{u}_{\mathrm{e}}(\mathrm{z}) \quad \mathrm{w}=\mathrm{w}_{\mathrm{e}}(\mathrm{z}) \quad \mathrm{T}=\mathrm{T}_{\mathrm{e}}(\mathrm{z})
$$

Here the superscript prime denotes the differential such as $h^{\prime}=d h / d x, \mathbf{n}$ is the unit vector in the normal direction, $\mathrm{T}_{*}$ is a constant reference temperature, and $\mathrm{k}$ and $\mathrm{H}$ are respectively the thermal conductivity of liquid and the heat transfer coefficient in the gas. Conditions (2.5)-(2.8) describerespectively the free surface $z=h(x)$ as a stream surface, the viscous stress in the tangent direction balancing with the thermocapillary force driven by the surface tension gradient, the momentum conservation in the normal direction, and the heat transfer across the free surface where the radiation effect is omitted. In addition to boundary conditions (2.4)-(2.9), the boundary conditions for the height of liquid layer should be given. And the problem of jet liquid film may, then, be solved.

Nondimensional quantities and parameters may be introduced on the basis of lubrication theory as follow: ${ }^{5}$

$$
\begin{gathered}
\xi=\frac{\mathrm{x}}{\mathrm{l}} \quad \xi=\frac{\mathrm{z}}{\mathrm{h}_{\mathrm{o}}} \quad \eta=\frac{\mathrm{h}}{\mathrm{h}_{\mathrm{o}}} \quad \epsilon=\frac{\mathrm{h}_{\mathrm{o}}}{\mathrm{l}} \\
\mathrm{U}=\frac{\mathrm{u}}{\mathrm{v}_{*}} \quad \epsilon \mathrm{W}=\frac{\mathrm{W}}{\mathrm{v}_{*}} \quad \epsilon^{2} \mathrm{P}=\frac{\mathrm{pl}}{\mu \mathrm{v}_{*}} \quad \Theta=\frac{\mathrm{T}}{\mathrm{T}_{*}}
\end{gathered}
$$

Here the typical temperature $T_{*}$ and the typical velocity $v_{*}$ are adopted respectively as

$$
\mathrm{T}_{*}=\mathrm{T}_{\mathrm{s}}(0) \quad \mathrm{v}_{*}=-\epsilon \sigma_{\mathrm{T}}{ }^{\top} \mathrm{T}_{*} / \rho v
$$

Thebasicfeature of thelubrication problem is that there are two typical scales of different orders of magnitude; that is, one of the typical scales I is much larger than the another $h_{0}$, and then, other quantities havedifferent orders of magnitude. Thenondimensional parameters in this case are the Reynolds number Re and Peclet number Pe:

$$
\mathrm{Re}=\frac{v_{*} \mathrm{I}}{v} \mathrm{Pe}=\frac{v_{*} \mathrm{I}}{\kappa}
$$

ThePrandtI number and M arangoni number may begiven as

$$
\mathrm{Re}=\operatorname{PrMa} \quad \mathrm{Ma}=-\sigma_{\mathrm{T}}{ }^{\top} \mathrm{T}_{*} \mathrm{l} / \kappa v=\epsilon \mathrm{Pe}
$$

Nondimensional equations and boundary conditions may then be written as

$$
\begin{gathered}
\frac{\partial U}{\partial \xi}+\frac{\partial W}{\partial \zeta}=0 \\
\operatorname{Re} \epsilon^{2}\left(U \frac{\partial U}{\partial \xi}+W \frac{\partial U}{\partial \xi}\right)=-\frac{\partial P}{\partial \xi}+\frac{\partial^{2} U}{\partial \xi^{2}}+\epsilon^{2 \frac{\partial^{2} U}{\partial \xi^{2}}} \\
\operatorname{Re} \epsilon^{4}\left(U \frac{\partial W}{\partial \xi}+W \frac{\partial W}{\partial \xi}\right)=-\frac{\partial P}{\partial \xi}+\epsilon^{2} \frac{\partial^{2} W}{\partial \xi^{2}}+\epsilon^{4 \frac{\partial^{2} W}{\partial \xi^{2}}} \\
P e \epsilon^{2}\left(U \frac{\partial \Theta}{\partial \xi}+W \frac{\partial \Theta}{\partial \xi}\right)=\frac{\partial^{2} \Theta}{\partial \xi^{2}}+\epsilon^{2 \frac{\partial^{2} \Theta}{\partial \xi^{2}}}
\end{gathered}
$$

and the boundary conditions are listed as follows: 
$\zeta=0$ :

$$
U=U_{0} \text { (const) } \quad W=0 \quad \Theta=\Theta_{s}(\xi)
$$

$\zeta=\eta(\xi):$

$$
\begin{aligned}
& \eta(0)=1 \\
& \mathrm{~W}(\xi, \eta)=\eta^{\prime} \mathrm{U}(\xi, \eta) \\
& \frac{\partial \mathrm{U}}{\partial \xi}+\epsilon^{2}\left[\left(\frac{\partial \mathrm{W}}{\partial \xi}-\eta^{\prime 2} \frac{\partial \mathrm{U}}{\partial \xi}\right)+2 \eta^{\prime}\left(\frac{\partial \mathrm{W}}{\partial \xi}-\frac{\partial \mathrm{U}}{\partial \xi}\right)-\epsilon^{2} \eta^{\prime} \frac{\partial \mathrm{W}}{\partial \xi}\right]= \\
& \sqrt{1+\epsilon^{2} \eta^{\prime 2}}\left(\frac{\partial \Theta}{\partial \xi}+\eta^{\prime} \frac{\partial \Theta}{\partial \xi}\right) \\
& \mathrm{P}=-\frac{\epsilon^{2}}{\mathrm{C}} \frac{\eta^{\prime \prime}}{\left(1+\epsilon^{2} \eta^{\prime 2}\right)^{3 / 2}}+\frac{2 \epsilon^{2}}{1+\epsilon^{2} \eta^{\prime 2}}\left[\left(\frac{\partial \mathrm{W}}{\partial \zeta}-\eta^{\prime} \frac{\partial \mathrm{U}}{\partial \zeta}\right)+\right. \\
& \left.\epsilon^{2} \eta^{\prime}\left(-\frac{\partial \mathrm{W}}{\partial \xi}+\eta^{\prime} \frac{\partial \mathrm{U}}{\partial \xi}\right)\right] \\
& \frac{\partial \Theta}{\partial \xi}-\epsilon^{2} \eta^{\prime} \frac{\partial \Theta}{\partial \xi}=-\operatorname{Bi}\left(\Theta-\Theta_{g}\right)\left(1+\epsilon^{2} \eta^{\prime 2}\right)^{1 / 2} \\
& \xi=0 \text { : } \\
& U=U_{e}(\zeta) \quad W=W_{e}(\zeta) \quad \Theta=\Theta_{e}(\zeta)
\end{aligned}
$$

wheretheCapillary number $\mathbf{C}=-\sigma_{\top}^{\prime} \mathbf{T}_{*} / \sigma_{0}$ is usually much smaller than unity and the nondimensional Boit number is defined by $\mathrm{Bi}=\mathrm{Hh}_{\mathrm{d}} / \mathrm{k}$.

\section{Perturbation Method}

Thenondimensional equations and boundary conditions show the relationships of the orders of magnitude, and the perturbation method can beapplied by the expansion based on the small parameter $\epsilon$. Due to the order of magnitude analysis (OMA), it requires that

$$
\mathrm{Re}=\mathrm{O}(1) \quad \mathrm{Pe}=\mathrm{O}(1) \quad \mathrm{Bi}=\mathrm{O}(1) \quad \mathrm{C}=\epsilon^{2} / \alpha=\mathrm{O}\left(\epsilon^{2}\right)
$$

where $\alpha$ is a constant. It is noted that definitions of the Reynolds number and the Peclet number in (2.13) are $\epsilon$ times smaller than the usual definitions, because the typical velocity $v_{*}$ is $\epsilon$ times smaller than the usual typical ther mocapillary vel ocity. Thequantities are expanded as follows:

$$
\begin{array}{r}
U=\sum_{n=0}^{\infty} \epsilon^{n} U^{(n)} \quad W=\sum_{n=0}^{\infty} \epsilon^{n} W^{(n)} \quad \Theta=\sum_{n=0}^{\infty} \epsilon^{n} \Theta^{(n)} \\
\eta=\sum_{n=0}^{\infty} \epsilon^{n} \eta^{(n)}
\end{array}
$$

If one substitutes relations (3.2) into the equations and boundary conditions, the problem can be solved order by order.

The zero's order relationships can be written as

$$
\begin{gathered}
\frac{\partial \mathbf{U}^{(0)}}{\partial \xi}+\frac{\partial \mathbf{W}^{(0)}}{\partial \xi}=0 \\
\frac{\partial \mathbf{P}^{(0)}}{\partial \xi}=\frac{\partial^{2} \mathbf{U}^{(0)}}{\partial \xi^{2}} \\
\frac{\partial \mathbf{P}^{(0)}}{\partial \zeta}=0 \\
\frac{\partial^{2} \Theta^{(0)}}{\partial \xi^{2}}=0
\end{gathered}
$$

and the boundary conditions are

$\zeta=0$ :

$$
\mathrm{U}^{(0)}=U_{\circ} \quad \mathrm{W}^{(0)}=0 \quad \Theta^{(0)}=\Theta_{\mathrm{s}}(\xi)
$$

$\zeta=\eta^{(0)}$

$$
\begin{gathered}
W^{(0)}=U^{(0)} \eta^{(0)} \\
\frac{\partial U^{(0)}}{\partial \xi}=-\frac{\partial \Theta^{(0)}}{\partial \xi}-\frac{d \eta^{(0)}}{d \xi} \frac{\partial \Theta^{(0)}}{\partial \xi} \\
P^{(0)}=-\alpha \frac{d^{2} \eta^{(0)}}{d \xi^{2}}
\end{gathered}
$$$$
\frac{\partial \Theta^{(0)}}{\partial \zeta}=-\operatorname{Bi}\left(\Theta^{(0)}-\Theta_{g}\right)
$$

$$
\xi=0:
$$

$$
\mathrm{U}^{(0)}=\mathrm{U}_{\mathrm{e}}(\zeta) \quad \mathrm{W}^{(0)}=\mathrm{W}_{\mathrm{e}}(\zeta) \quad \Theta^{(0)}=\Theta_{\mathrm{e}}(\zeta) \quad \eta^{(0)}=\frac{1}{(3.12)}
$$

$\xi=1:$

$$
\eta^{(0)}=\eta_{\mathrm{m}}
$$

where $\eta_{\mathrm{m}}$ is the height at the cross section $\mathrm{z}=1$ and is adopted typically as $\eta_{\mathrm{m}}=1$. Basic equations (3.3)-(3.6) of the zero's order of magnitude are similar to the ones given in refs 4 and 5.

Equation (3.6) means that the temperature is a linear function of $\xi$ and may be obtained as follows by using boundary condition (3.11):

$$
\Theta^{(0)}(\xi, \zeta)=\Theta_{\mathrm{s}}-\frac{\operatorname{Bi}\left(\Theta_{\mathrm{s}}-\Theta_{\mathrm{g}}\right)}{1+\mathrm{Bi} \eta^{(0)}} \zeta
$$

Relationship (3.14) shows that the heat transfers from the solid of temperature $\Theta_{s}$ to the free surface of temperature $\Theta^{(0)}\left(\xi, \eta^{(0)}\right)$ and then to the gas of temperature $\Theta_{\mathrm{g}}$. The temperature in the liquid film will be uniform if thesolid boundary $\xi=0$ keeps a uniform temperature the sameas the environmental gas temperature. I $\mathrm{n}$ this case, there will be no thermocapillary flow in the liquid layer.

Equation (3.5) implies that the pressure is a function of $\xi$ only, and then condition (3.10) at the free surface gives

$$
P^{(0)}(\xi)=-\alpha \frac{d^{2} \eta^{(0)}}{d \xi^{2}}
$$

Substituting (3.15) intoequations (3.3) and (3.4) and using free surface condition (3.8), the vel ocity fiel $d$ in the liquid layer may be written as

$$
\begin{gathered}
U^{(0)}=-\frac{\alpha}{2} \frac{d^{3} \eta^{(0)}}{d \xi^{3}} \xi^{2}+A(\xi) \zeta+U_{\circ} \\
W^{(0)}=\frac{\alpha}{6} \frac{d^{4} \eta^{(0)}}{d \xi^{4}} \zeta^{3}-\frac{1}{2} \frac{d A}{d \xi} \xi^{2}
\end{gathered}
$$

and the function $A$ is

$$
\mathrm{A}(\xi)=\alpha \eta^{(0)} \frac{\mathrm{d}^{3} \eta^{(0)}}{\mathrm{d} \xi^{3}}-\frac{\mathrm{d}}{\mathrm{d} \xi}\left[\Theta_{\mathrm{s}}-\frac{\mathrm{Bi}\left(\Theta_{\mathrm{s}}-\Theta_{\mathrm{g}}\right)}{1+\mathrm{Bi} \eta^{(0)}} \eta^{(0)}\right]
$$

Relationship (3.16) shows that longitude velocity consists of a main jet vel ocity $U_{0}$ in addition tothecontribution 
of the cross-section variation and the thermocapillary effect. By using solutions (3.16) and (3.17), a stream function $\Psi(\xi, \xi)$ may be easily obtained as

$$
\begin{aligned}
& \Psi(\xi, \zeta)=\frac{\alpha}{6} \frac{d^{3} \eta^{(0)}}{d \xi^{3}}\left(3 \eta^{(0)}-\xi\right) \xi^{2}- \\
& \frac{1}{2} \frac{d}{d \xi}\left[\Theta_{s}-\frac{B i\left(\Theta_{s}-\Theta_{g}\right)}{1+B i \eta^{(0)}} \eta^{(0)}\right] \zeta^{2}+U_{o} \xi+\Psi_{\circ}
\end{aligned}
$$

where $\Psi_{\mathrm{o}}$ is an integral constant and may be selected as zero. Solutions (3.14)-(3.17) depend on the height of the liquid layer, which equation is determined by boundary condition (3.9).

The velocity and temperature boundary conditions in (3.12) and (3.13) cannot be given arbitrarily, and must satisfy the distributions of general solutions (3.14), (3.16), and (3.17).

\section{Height Equation and Its Solution}

By substitution of sol ution (3.14), (3.16), and (3.17) into boundary condition (3.9) at the free surface, the height equation of the liquid layer is demonstrated as

$$
\frac{\mathrm{d}}{\mathrm{d} \xi}\left(\frac{\alpha}{6} \eta^{(0)^{3}} \frac{\mathrm{d}^{3} \eta^{(0)}}{\mathrm{d} \xi^{3}}-\frac{\mathrm{A}(\xi)}{2} \eta^{(0)^{2}}-\mathrm{U}_{\circ} \eta^{(0)}\right)=0
$$

Theheight equation is a fourth-order ordinary differential equation, and its solution needs four boundary conditions on the height. The initial integration of eq (4.1) gives a third-order ordinary differential equation including an integral constant.

$$
\begin{aligned}
& \frac{\mathrm{d}^{3} \eta^{(0)}}{\mathrm{d} \xi^{3}}=\frac{3}{2 \alpha \eta^{(0)}\left(1+\mathrm{Bi} \eta^{(0)}\right)}\left[\frac{\mathrm{d} \Theta_{\mathrm{s}}}{\mathrm{d} \xi}-\frac{\mathrm{Bi}\left(\Theta_{\mathrm{s}}-\Theta_{\mathrm{g}}\right)}{1+\mathrm{Bi} \eta^{(0)}} \frac{\mathrm{d} \eta^{(0)}}{\mathrm{d} \xi}\right]- \\
& \frac{3 \mathrm{U}_{\mathrm{o}}}{\alpha} \frac{1}{\eta^{(0)^{2}}}+\frac{\mathrm{C}_{1}}{\eta^{(0)^{3}}}
\end{aligned}
$$

where integral constant $c_{1}$ may be given by the boundary condition at $\xi=0$ :

$$
\begin{aligned}
c_{1}= & \frac{3 U_{o}}{\alpha}+\frac{d^{3} \eta(0)}{d \xi^{3}}- \\
& \quad \frac{3}{2 \alpha(1+B i)}\left[\frac{d \Theta_{s}(0)}{d \xi}-\frac{B i\left(1-\Theta_{g}\right)}{1+B i} \frac{d \eta^{(0)}(0)}{d \xi}\right],
\end{aligned}
$$

Herethe boundary conditions $\eta^{(0)}(0)=1$ and $\Theta_{s}(0)=1$ are used.

Four boundary conditions of the liquid layer height at the exit of the melt vessel $\xi=0$ and at the longitudinal exit cross section $\xi=1$ may besel ected by different options and then give different distributions of the height. In the present paper, the boundary conditions are given as follows:

$$
\eta^{(0)}(0)=1 \quad \frac{\mathrm{d} \eta^{(0)}(0)}{\mathrm{d} \xi}=\gamma \quad \frac{\mathrm{d}^{2} \eta^{(0)}(0)}{\mathrm{d} \xi^{2}}=\beta \quad \eta^{(0)}(1)=1
$$

Here $\gamma$ is the expansion angle of the jet and $\beta$ is the inclination variation of the expanding angle at the exit of melt vessel. Different selections of $(\gamma, \beta)$ on the variation of height areanalyzed for understanding themain feature of the thermocapillary effect in the jet liquid film. The first and last conditions in (4.5) require that the cross sections are the same at the exit of melt vessel $\xi=0$ and

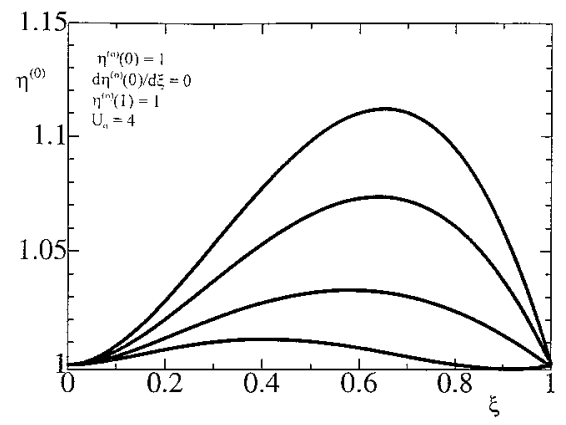

Figure 2. Influence of the expending angle gradients on the height profiles of the jet liquid layer for cases $\eta^{(0)}(0)=1, \mathrm{~d} \eta^{(0)}$ $(0) / \mathrm{d} \xi=0, \eta^{(0)}(1)=1$, and $\mathrm{U}_{0}=4$. The profiles relateto $\beta=0.7$, $1.0,1.5$, and 2.0 respectively from bottom to top.

at the longitudinal exit position $\xi=1$. The last condition in (4.5) is reasonable, and relates to a specified value of $\mathrm{d}^{3} \eta(0) / \mathrm{d} \xi^{3}$. This condition has no limitation in discussing the general features and may beeasily changed to a given value, which may be designed by the engineering consideration.

The temperature $\Theta_{s}(\xi)$ on the solid boundary $\xi=0$ should be obtained by analyzing the heat transfer process between liquid layer, solid film, and environmental gas, and this process is coupled with the process in the liquid layer. For simplification, the temperature at the solid boundary will be given, for example, as follows:

$$
\Theta_{\mathrm{s}}=1-\left(1-\Theta_{l}\right) \frac{1-\exp (-\mathrm{n} \xi)}{1-\exp (-\mathrm{n})}
$$

Here $1 /$ n denotes a typical scale of heat transfer. The heat transfer and thetemperature decreaseare relatively large in the region near the vessel exit and then becomesl ower afterward if the melt temperature $T_{0}$ at the vessel exit is larger than the environmental gas temperature $T_{g}$ and the temperature $T_{\mid}$at $x=I$. Distribution (4.6) shows that the temperature on the solid film changes from the exit value $T_{0}$ at $x=0$ to the temperature $T_{1}$ at $x=I$, and the most important change appears in the region $0 \leq x \leq 1 / n$.

Substituting temperature distribution (4.6) into height eq (4.4), thedetermined solution of eq (4.4) under boundary conditions (4.5) may then beobtained. Typical parameters in eq (4.4) are adopted as

$$
\alpha=0.5 \quad \Theta_{g}=0.1 \quad \Theta_{1}=0.1 \quad \mathrm{Bi}=0.5 \quad \mathrm{n}=5
$$

and, then, the equation is reduced as

$$
\begin{aligned}
\frac{\mathrm{d}^{3} \eta^{(0)}}{\mathrm{d} \xi^{3}}=\frac{\mathrm{c}_{1}}{\eta^{(0)^{3}}}- & \frac{6 \mathrm{U}_{\mathrm{o}}}{\eta^{(0)^{2}}}- \\
& {\left[\frac{\mathrm{ne} \mathrm{e}^{-\mathrm{n} \xi}}{1-\mathrm{e}^{-\mathrm{n}}}+\frac{1}{2+\eta^{(0)}} \frac{\mathrm{e}^{-\mathrm{n} \xi}-\mathrm{e}^{-\mathrm{n}}}{1-\mathrm{e}^{-\mathrm{n}}} \frac{\mathrm{d} \eta^{(0)}}{\mathrm{d} \xi}\right] }
\end{aligned}
$$

where the integral constant $c_{1}$ is determined by the last condition of (4.5).

Ordinary differential eq (4.8) under the boundary condition and parameter conditions is solved by using the Runge-Kutta method, and the last condition of (4.5) is satisfied by an interaction process. The cases of zero expandingangle $\gamma=0$ areinteresting sometimes. In these cases, the influence of gradient of expanding angle on the height distribution is shown in Figure 2 for $U_{0}=4$. The larger the gradient of expanding angle at the vessel exit, thelarger the height in thejet film. In the case of thezero 


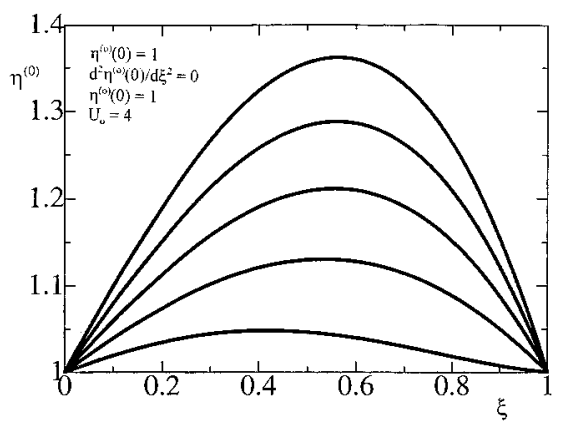

Figure 3. Influence of the expending angles on the height profiles of the jet liquid layer for cases $\eta^{(0)}(0)=1, \mathrm{~d}^{2} \eta^{(0)}(0) / \mathrm{d} \xi^{2}$ $=0, \eta^{(0)}(1)=1$, and $U_{0}=4$. The profiles relate to $\gamma=0.2,0.4$, $0.6,0.8$, and 1.0 respectively from bottom to top.

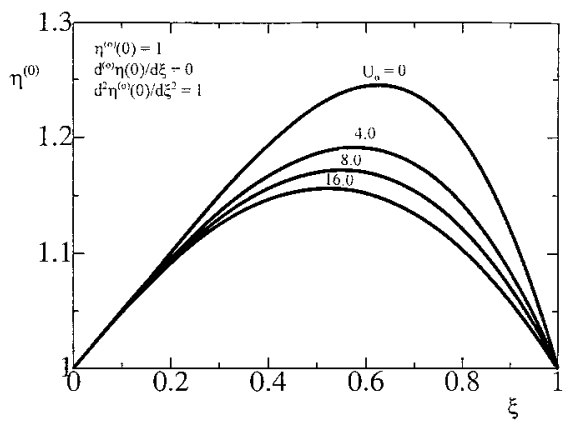

Figure 4. Influence of jet velocities on the height profiles of the jet liquid layer for case $\eta^{(0)}(0)=1, \mathrm{~d} \eta^{(0)}(0) / \mathrm{d} \xi=0, \mathrm{~d}^{2} \eta^{(0)}$. $(0) / \mathrm{d} \xi^{2}=1.0$, and $\eta^{(0)}(1)=1$. The profiles relate to $U_{0}=0,4.0$, 8.0 , and 16.0 respectively from top to bottom.

gradient of expanding angle $\beta=0$, the influence of expanding angle $\gamma$ at the vessel exit is given in Figure 3 . The larger the expanding angle $\gamma$, the larger the height. The results show that the influence of expanding angle is relatively moredirect and important in comparison with the one of the gradient of expending angle. The influence of jet velocity $U_{0}$ is summarized in Figure 4 for the case $\gamma=0$ and $\beta=1.0$. There is only thermocapillary flow and no jet flow in the special case of zero jet velocity $U_{0}=0$, and the liquid film with thermocapillary flow persists on a motionless solid film.

With substitution of boundary temperature distribution (4.6) into the temperature distribution (3.14), the temperature in the jet liquid layer may be demonstrated as

$$
\begin{aligned}
& \Theta^{(0)}(\xi, \xi)=1-\left(1-\Theta_{\mid}\right) \frac{1-e^{-n \xi}}{1-e^{-n}}- \\
& \frac{\operatorname{Bi}\left[\left(1-\Theta_{g}\right)\left(1-e^{-n}\right)-\left(1-\Theta_{\mid}\right)\left(1-e^{-n \xi}\right)\right.}{\left(1+B i \eta^{(0)}\right)\left(1-e^{-n}\right)} \zeta
\end{aligned}
$$

Thefirst twoterms on theright-hand sideareindependent of $\xi$, and the third term is a linear function of $\xi$. On the basis of the solutions of liquid layer height, if

$$
\eta^{(0)}(\xi)=1+\delta(\xi)
$$

where $\delta$ is a small quantity and satisfies the relation in order of magnitude $\delta=\mathrm{O}(\epsilon)$. By using parameters adoptions (4.8) and taking $U=4$ and $n=5$, the equation of temperature distribution (4.9) is reduced as

$$
\begin{aligned}
& \Theta^{(0)}(\xi, \zeta)=\left(0.093895+0.906105 \mathrm{e}^{-5 \xi}\right)- \\
& \left(0.302035 \mathrm{e}^{-5 \xi}-0.002035\right)(1-\delta) \xi+\mathrm{O}\left(\delta^{2}\right)
\end{aligned}
$$

Relationship (4.11) gives a linear temperature distribution at the vessel exit $\Theta^{(0)}(0, \xi)=1-0.3 \xi$ and a uniform temperature at the longitudinal exit cross section $\Theta^{(0)}(1, \zeta)=0.1$. These are the reasonable boundary conditions required by (3.12) and (3.13). The temperature on the free surface is obtained as

$$
\Theta^{(0)}\left(\xi, \eta^{(0)}(\xi)\right)=0.095930+0.604070 \mathrm{e}^{-5 \xi}+\mathrm{O}\left(\delta^{2}\right)
$$

The nondimensional temperature on the free surface is mainly dependent on the heat transfer process decreasing from a value 0.7 at the vessel exit $\xi=0$ to a value 0.1 at thesol idification cross section $\xi=1$ and is decoupled from the jet flow in an accuracy of $\mathrm{O}\left(\delta^{2}\right)$.

Similar discussions may be applied to the flow fields, which depend mainly on both thermocapillary effect and the jet flow. By using height eq (4.8), the equation of the stream function (3.19) is reduced to

$$
\begin{aligned}
& \Psi^{(0)}(\xi, \zeta)= \frac{\left(\eta^{(0)}-\xi\right) \xi^{2}}{4 \eta^{(0)}\left(1+\mathrm{Bi} \eta^{(0)}\right)} \frac{\mathrm{d} \Theta_{\mathrm{s}}}{\mathrm{d} \xi}- \\
& \frac{\mathrm{Bi}\left(\Theta_{\mathrm{s}}-\Theta_{\mathrm{g}}\right)\left(\eta^{(0)}-\xi\right) \xi^{2}}{4 \eta^{(0)}\left(1+\mathrm{Bi} \eta^{(0)}\right)^{2}} \frac{\mathrm{d} \eta^{(0)}}{\mathrm{d} \xi}+ \\
& \frac{\mathrm{U}_{0}\left(2 \eta^{(0)}-\zeta\right)\left(\eta^{(0)}-\xi\right) \xi}{2 \eta^{(0)}}+\frac{\mathrm{C}_{1} \alpha\left(3 \eta^{(0)}-\zeta\right) \xi^{2}}{6 \eta^{(0)^{3}}}
\end{aligned}
$$

where the integral constant $\Psi_{\mathrm{o}}$ in relationship (3.19) is adopted as zero. The stream functions $\Psi(\xi, 0)=0$ and $\Psi\left(\xi, \eta^{(0)}(\xi)\right)=\mathrm{c}_{1} \alpha / 3$ (constant) correspond to that both the solid boundary $\xi=0$ and the free surface $\xi=\eta^{(0)}(\xi)$ are stream surfaces. With substitution of parameters (4.5) and expression (4.6), the stream function is given as

$$
\begin{gathered}
\Psi^{(0)}(\xi, \zeta)=-4.530527 e^{-5 \xi} \frac{\left(\eta^{(0)}-\xi\right) \xi^{2}}{\eta^{(0)}\left(2+\eta^{(0)}\right)^{2}}+ \\
\left(0.453053 e^{-5 \xi}-0.003052\right) \frac{\left(\eta^{(0)}-\xi\right) \xi^{2}}{\eta^{(0)}\left(2+\eta^{(0)}\right)^{2}} \frac{\mathrm{d} \eta^{(0)}}{\mathrm{d} \xi}+ \\
\frac{\mathrm{U}_{0}\left(2 \eta^{(0)}-\zeta\right)\left(\eta^{(0)}-\zeta\right) \xi}{2 \eta^{(0)}}+\frac{\mathrm{c}_{1}\left(3 \eta^{(0)}-\zeta\right) \xi^{2}}{12 \eta^{(0)^{3}}}
\end{gathered}
$$

In considerations of relationship (4.10) and the small variation of liquid layer height $\left|\mathrm{d} \eta^{(0)} / \mathrm{d} \xi\right|=\mathrm{O}(\delta)$, thestream function may be expanded as

$$
\begin{array}{r}
\Psi^{(0)}(\xi, \zeta)=-0.503363 \mathrm{e}^{-5 \xi}(1-\xi) \xi^{2}+\frac{\mathrm{U}_{\mathrm{o}}}{2}(2-\xi)(1- \\
\zeta) \zeta+\frac{\mathrm{c}_{1}}{12}(3-\xi) \xi^{2}+\left\{\left(0.05034 \mathrm{e}^{-5 \xi}-0.00339\right)(1-\right. \\
\zeta) \xi^{2} \frac{\mathrm{d} \eta^{(0)}}{\mathrm{d} \xi}+\left[0.167788 \mathrm{e}^{-5 \xi}(2-5 \xi) \xi^{2}+\frac{\mathrm{U}_{\mathrm{o}}}{2}(1-\zeta)(2+\right. \\
\left.\left.\xi)+\frac{\mathrm{C}_{1}}{4}(\xi-2)\right] \delta\right\}+\mathrm{O}\left(\delta^{2}\right)
\end{array}
$$

The field distributions may be calculated directly from eq (4.14), and Figure 5 gives an example. 


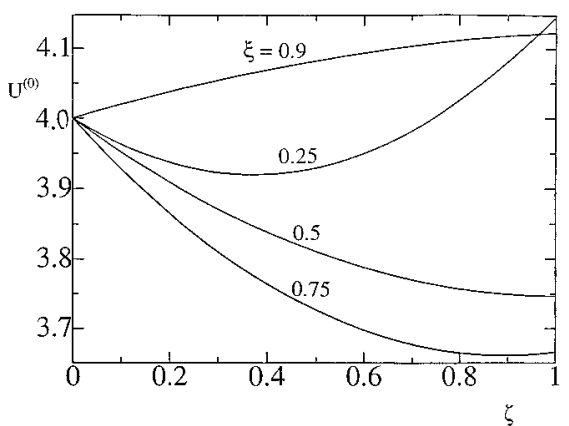

Figure 5. Velocity profiles in the jet liquid layer for case $\eta^{(0)}(0)$ $=1, \mathrm{~d} \eta^{(0)}(0) / \mathrm{d} \xi=0.5, \mathrm{~d}^{2} \eta^{(0)}(0) / \mathrm{d} \xi^{2}=0, \eta^{(0)}(1)=1$, and $\mathrm{U}_{\mathrm{o}}=4$.

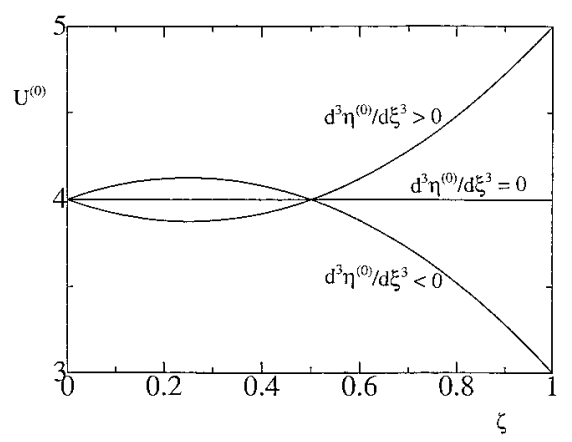

Figure 6. Typical velocity profiles in the downward region of jet liquid film.

By substitution of relationships (4.6) and (4.7), relationship (3.16) for the velocity component in the jet direction may be written as

$$
\begin{gathered}
\mathrm{U}^{(0)}=\mathrm{U}_{\mathrm{o}}+\mathrm{a} \xi-\mathrm{b} \xi^{2} \\
\alpha=\left[\frac{\eta^{(0)}}{2} \frac{\mathrm{d}^{3} \eta^{(0)}}{\mathrm{d} \xi^{2}}+\frac{9.061052}{2+\eta^{(0)}} \mathrm{e}^{-5 \xi}+\right. \\
\left.\left(\mathrm{e}^{-5 \xi}-\mathrm{e}^{-5}\right) \frac{1.812211}{\left(2+\eta^{(0)}\right)^{2}} \frac{\mathrm{d} \eta^{(0)}}{\mathrm{d} \xi}\right] \quad \mathrm{b}=\frac{1}{4} \frac{\mathrm{d}^{3} \eta^{(0)}}{\mathrm{d} \xi^{3}}
\end{gathered}
$$

where the values of $\eta^{(0)}, d \eta^{(0)} / d \xi$, and $d^{3} \eta^{(0)} / d \xi^{3}$ are given by the solution of height eq (4.8). Then, the velocity component as a second-order function of $\xi$ may beobtained, as an examplein $\mathrm{F}$ igure 5, which gives the vel ocity profiles at cross section $\xi=2.5,0.5,0.75$, and 0.9 for the case $\gamma$ $=0.5, \beta=0$, and $U_{0}=4$. The results show that a return flow is induced by the thermocapillary effect and crosssection variation in addition tothe jet flow $U_{0}$ in theregion near the exit of vessel. The values of $a$ and $b$ are listed as follows:

$$
\begin{array}{llllll}
\xi & 0 & 0.25 & 0.50 & 0.75 & 0.90 \\
\text { a } & -0.006323 & -0.428667 & -0.759072 & -0.503546 & 0.203776 \\
\text { b } & 1.557014 & 0.575915 & 0.425068 & 0.250218 & -0.080106
\end{array}
$$

It could be seen that the last twoterms in the bracket are relatively smaller in the downward region of jet, and the velocity component may be approximately written as

$$
\mathrm{U}^{(0)}(\xi, \zeta) \cong \mathrm{U}_{\mathrm{o}}+\frac{2 \eta^{(0)}-\xi}{4} \frac{\mathrm{d}^{3} \eta^{(0)}(\xi)}{\mathrm{d} \xi^{3}} \zeta \quad \xi>0.5
$$

The typical velocity profiles are show in Figure 6.

If the temperature difference between $T_{e}$ at the vessel exit $\mathrm{x}=0$ and $\mathrm{T}_{\mathrm{g}}$ of the environmental gas or the temperature $T_{1}$ at $x=I$ is not large enough, the temper-

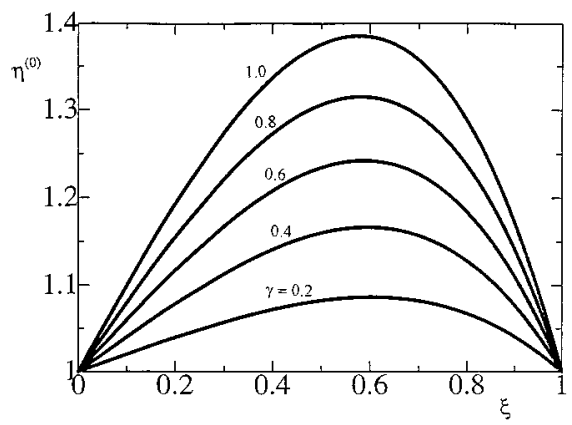

Figure 7. I nfluence of expending angles on the height profiles of the jet liquid layer for a linear temperature distribution (4.9) at the solid boundary and $\eta^{(0)}(0)=1, \mathrm{~d}^{2} \eta^{(0)}(0) / \mathrm{d} \xi^{2}=0$, and $\eta^{(0)}(1)$ $=1$.

ature distribution $T_{s}$ is better to be described approximately by a linear function of $x$ such as

$$
\Theta_{\mathrm{s}}=1-\left(1-\Theta_{1}\right) \xi
$$

Substituting relationship (4.18) into height eq (4.2) gives

$$
\begin{aligned}
& \frac{\mathrm{d}^{3} \eta^{(0)}}{\mathrm{d} \xi^{3}}=\frac{\mathrm{c}_{1}}{\eta^{(0)^{3}}}-\frac{6 \mathrm{U}_{\circ}}{\eta^{(0)^{2}}}-5.4\left[\frac{1}{\left(2+\eta^{(0)}\right) \eta^{(0)}}+\right. \\
&\left.\frac{1-\xi}{\left(2+\eta^{(0)}\right)^{2} \eta^{(0)}} \frac{\mathrm{d} \eta^{(0)}}{\mathrm{d} \xi}\right]
\end{aligned}
$$

Ordinary differential eq (4.19) can be solved under boundary conditions (4.5) and parameter conditions (4.7), and similar conclusions are obtained. As an example, Figure 7 gives the influence of the expanding angle on the height distributions in the case of $U_{0}=4$ for temperature distribution (4.18). The heights of the jet liquid film are relatively larger, and their maximums shift relatively into the downward direction of the jet in the case of linear temperature distributions (4.18) in comparison with the results of Figure 3 for temperature distributions (4.6).

\section{Discussion}

The lubrication approximation and the perturbation method are applied to discuss the problem of jet liquid film, and theanalytical solutions of temperature, pressure, and velocity depending on the height of liquid film were obtained. The determined problem of the liquid height is described by a third-order ordinary differential equation with an integral constant, and 4 boundary conditions of liquid height are required to determine the 4 integral constants. The problem of liquid height was solved by using the Runge-Kutta method, and then, the temperature, pressure, and velocity fields in the jet liquid film were obtained. The results show that the influence of thermocapillary effect may enlarge the cross section of the jet liquid. The influence of typical parameters on the height distribution is discussed specifically.

It should benoted that theapproximations of lubrication theory and perturbation theory have some limitations. The Barus effect has a larger variation of cross section, which may be two or three times, and the region of the enlarging cross section is cl oser tothevessel exit. However, the assumption of last relationship in (3.1) permits only a small variation of cross section. Therefore, the results of this paper show mainly the mechanism of the cross section variation due to the thermocapillary effect and jet flow. The larger variation of cross section needs to be studied by omitting theassumption and should bestudied in the future. 
Newtonian fluid is assumed in this paper. Thermocapillary flow may increase the cross section of the liquid layer on the basis of this assumption. This conclusion does not imply that the rheology of the liquid layer is not important. In fact, moreattention should be paid to study the rheology, the manufacture model, and the detailed parameter region if the fiber processing is considered. However, the main purpose of this paper is concentrated in the mechanism study, which shows that the heat transfer may induce thermocapillary flow, which will increase the cross section even for a Newtonian fluid. An experiment tocheck theresults of this paper is important. Usually, the thermocapillary effect will be coupled with the effect of rheological properties during the die swell, and both effects depend on the temperature gradient. However, the thermocapillary effect may be nearly distinguished in an experiment from the effect of the rheological effect if either the experimental condition is limited in the initial Newtonian region, and second Newtonian region ${ }^{8}$ or a modeling medium of Newtonian fluid is adopted as the experimental medium. Morestudies should be initiated, especially in connecting with the experimental studies and the manufacture processing.

Acknowledgment. W.R.H. acknowledges the kind invitation of Prof. Y. Sugioka, President of Kyushu University, and the kind hospitality of Prof. N. I maishi of the same university enabling him to be a visiting Professor at the Institute of Advanced Material Study of Kyushu U ni versity. This research was completed during the visiting period.

\section{LA991563V}

(8) Lenk, R. S. Polymer Rheol ogy; Applied Science Publishers Ltd.: London, 1978; Chapter 1. 